\title{
Editorial
}

\section{Liver disease of uncertain cause}

\author{
Bone Marrow Transplantation (2004) 33, 977-978. \\ doi:10.1038/sj.bmt.1704464
}

When I began to study the clinical epidemiology of liver injury after myeloablative conditioning regimens and hematopoietic cell transplant, it was apparent that about $10-20 \%$ of patients who evinced liver dysfunction post transplant could not be readily classified as to etiology. ${ }^{1,2}$ For research purposes, I created a separate category for these patients, Liver Disease of Uncertain Cause.

The case reported by Favre et al in this issue is reminiscent of my experiences in dealing with diagnostic uncertainty when patients develop liver problems after transplant. Their patients developed elevated serum ALT at day 13 and mild hyperbilirubinemia and hepatomegaly at day 15 after a myeloblative regimen containing cyclophosphamide, total body irradiation, and thioTEPA. When serum ALT levels exceeded $1000 \mathrm{U} / 1$, the authors made a clinical diagnosis of acute hepatitis $\mathrm{B}$ because of the donor's HBV antibody status and the patient's pre-existing HBV infection. Treatment was started with horse antilymphocyte globulin and mycophenolate mofetil to blunt HBV-specific donor T-cell activity in the liver. Over the ensuing weeks, the patient's liver returned to normal and she ultimately seroconverted to anti-HBs.

When I reviewed this paper, I was skeptical that acute hepatitis B was the diagnosis, for the following reasons: (1) the patient had been started on lamivudine before the transplant (a highly effective strategy to prevent HBVrelated liver damage after $\mathrm{HCT}$ ) and there was insignificant viral replication after transplant, as judged by serum HBV DNA levels; (2) the patient's liver disease began before substantive engraftment of donor cells; (3) the sudden onset of hepatomegaly seemed out of proportion to the degree of viral replication, the paucity of donor T-cells, and ongoing immunosuppressive therapy; and (4) there was no proof that HBV-antigen-reactive donor T-cells were present in the patient. ${ }^{3}$ From my disadvantageous perch thousands of miles away from the bedside, my first hypothesis would have been that conditioning therapy had caused sinusoidal liver injury. Three liver-toxic drugs had been used in the conditioning regimen in high doses, and the somewhat later appearance of liver injury is typical of thioTEPA-related sinusoidal damage (sinusoidal obstruction syndrome, also known as venocclusive disease of the liver). ${ }^{4}$ When we examined the causes of extreme elevation of serum ALT among 2136 consecutive patients who were post transplant, the leading cause was SOS, with peak ALT values at day $23 \pm 9 .{ }^{5}$ This patient's peak ALT occurred at day 16. The cause of elevated ALT in patients with SOS is likely ischemia, as embolized sinusoidal endothelial cells and extensive deposition of collagen within sinusoids compromise perfusion of hepatocytes in zone 3 of the liver acinus. Thus, my initial diagnosis of this patient's liver disease would have been SOS. The authors do not think that SOS was likely because the published criteria for SOS were not met. As the author of the Seattle criteria for diagnosis of SOS, I can fairly point out that these criteria were established primarily for research purposes, that is, to have a high positive predictive value for toxic sinusoidal injury, as proven by liver histology. However, the spectrum of clinical signs and symptoms of sinusoidal injury is far wider, encompassing ALT elevations, isolated jaundice, isolated weight gain, gall bladder wall edema, and pain. When we examined autopsy liver tissue using a study design where the pathologist was blinded to clinical information, roughly half of the transplant patients whose liver dysfunction had been classified as Liver Disease of Uncertain Cause proved to have toxic sinusoidal injury despite failing to meet the published criteria for the diagnosis of SOS. ${ }^{6}$

What should be done in such cases to remove uncertainty? Should anti-T-cell therapy have been instituted in this case without a clear diagnosis? Had I been at the bedside, I would have suggested a transvenous procedure that measured the wedged hepatic venous pressure gradient and obtained liver tissue for histology. ${ }^{7,8}$ These procedures can be done safely even in thrombocytopenic transplant patients and provide prompt diagnostic information.

The point here is that uncertainty in clinical diagnosis is relatively common in dealing with liver disease after transplant. Also, Occam's Razor may be disposable in the transplant setting - that is, more than one pathophysiological process may be present at the same time. I have never regretted measuring the hepatic venous pressure gradient and looking at liver history in order to resolve uncertainty. As it turned out, this patient's liver disease improved after the institution of anti-T-cell therapy. Whether this therapy was causal in the resolution of liver disease, or whether attribution of recovery to therapy was 'post hoc, ergo propter hoc' reasoning, can never be known with certainty.

My research is supported by the following grants from the National Institutes of Health, National Cancer Institute: CA 15704 and CA 18029.

GB McDonald Gastroenterology/Hepatology Section,
Fred Hutchinson
Cancer Research Center and
University of Washington
School of Medicine,
Seattle, USA

\section{References}

1 McDonald GB, Hinds MS, Fisher LB et al. Venocclusive disease of the liver and multiorgan failure after bone marrow transplantation: a cohort study of 355 patients. Ann Intern Med 1993; 118: 255-267. 
2 McDonald GB, Slattery JT, Bouvier ME et al. Cyclophosphamide metabolism, liver toxicity, and mortality following hematopoietic stem cell transplantation. Blood 2003; 101: 2043.

3 Lau GK, Suri D, Liang R et al. Resolution of chronic hepatitis $\mathrm{B}$ and anti-HBs seroconversion in humans by adoptive transfer of immunity to hepatitis B core antigen. Gastroenterology 2002; 122: 614-624.

4 Lee JL, Gooley T, Bensinger W et al. Venocclusive disease of the liver after busulfan, melphalan, and thioTEPA conditioning therapy: incidence, risk factors, and outcome. Biol Blood Marrow Transplant 1999; 5: 306-315.

5 Strasser SI, McDonald SJ, Schoch HG et al. Severe hepatocellular injury after hematopoietic cell transplant: incidence and etiology in 2136 consecutive patients. Hepatology 2000; 32: $299 \mathrm{~A}$.

6 Shulman HM, Fisher LB, Schoch HG et al. Venocclusive disease of the liver after marrow transplantation: histologic correlates of clinical signs and symptoms. Hepatology 1994; 19: $1171-1180$.

7 Carreras E, Granena A, Navasa M et al. Transjugular liver biopsy in bone marrow transplantation. Bone Marrow Transplant 1993; 11: 21-26.

8 Shulman HM, Gooley T, Dudley MD et al. Utility of transvenous liver biopsies and wedged hepatic venous pressure measurements in sixty marrow transplant recipients. Transplantation 1995; 59: 1015-1022. 